\title{
DISTRIBUIÇÃO VOLUMÉTRICA E ESPECTRO DE GOTAS DE BICOS HIDRÁULICOS DE JATO PLANO DE FAIXA EXPANDIDA XR11003
}

\author{
FELIPE T. DA CAMARA ${ }^{1}$, JOSÉ L. SANTOS ${ }^{2}$, ELIAS A. SILVA ${ }^{3}$, \\ MARCELO DA C. FERREIRA ${ }^{4}$
}

\begin{abstract}
RESUMO: O presente trabalho teve por objetivos avaliar o espectro de gotas e os perfis de distribuição volumétrica de um bico hidráulico de jato plano de faixa expandida, modelo XR11003. Utilizou-se de analisador de partículas a laser para avaliar o espectro de gotas e de mesa de deposição para análise da distribuição volumétrica. O ensaio do espectro de gotas foi em delineamento inteiramente casualizado, em esquema fatorial $2 \times 2 \times 3$, com três repetições, em que o primeiro fator representa o líquido pulverizado ( $\mathrm{L} 1=$ água e $\mathrm{L} 2=$ água $+0,1 \%$ de espalhante adesivo não-iônico), o segundo representa a pressão de pulverização $(\mathrm{P} 1=200 \mathrm{kPa}$ e P2 $=400 \mathrm{kPa})$ e o terceiro representa três bicos hidráulicos XR11003 de jato plano (B1, B2 e B3). No ensaio de distribuição volumétrica, o delineamento foi o inteiramente casualizado, em esquema fatorial com dois fatores, não se avaliando o fator bico. Dos resultados do espectro de gotas, observaram-se maior tamanho de gotas para a pressão de $200 \mathrm{kPa}$ e menor amplitude relativa quando se utilizou $0,1 \%$ de adjuvante. Para os perfis de distribuição volumétrica, ocorreu aumento na faixa de deposição e no espaçamento entre bicos com C.V. de $10 \%$, com a adição de $0,1 \%$ de adjuvante e aumento na pressão.
\end{abstract}

PALAVRAS-CHAVE: surfactante, líquido de aplicação, tecnologia de aplicação.

\section{VOLUMETRIC DISTRIBUTION AND SPECTRUM OF DROPS OF HIDRAULIC NOZZLES OF PLANE JET OF EXPANDED STRIP XR11003}

\begin{abstract}
The present work had for objective to evaluate the spectrum of drops and the volumetric distribution of a hydraulic nozzle of a plane jet of expanded strip, model XR11003. A laser particle analyzer was used to evaluate the spectrum of drops and a deposition table was used for analysis of the volumetric distribution. The experiment of the spectrum of drops was a completely randomized design, factorial $2 \times 2 \times 3$, with three repetitions, where the first factor represents the sprayed liquid $(\mathrm{L} 1=$ water and $\mathrm{L} 2=$ water $+0,1 \%$ of no-ionic surfactant), the second factor represents the application pressure $(\mathrm{P} 1=200 \mathrm{kPa}$ e P2 $=400 \mathrm{kPa})$ and the third factor represents three nozzles XR11003 of the plane jet (B1, B2 e B3). The experiment of volumetric distribution was completely randomized design, factorial $2 \times 2$, not evaluating the factor nozzle. The results evidenced larger size of drops for the pressure of $200 \mathrm{kPa}$, and smaller relative width when it was used $0,1 \%$ of adjuvant. For the profiles of volumetric distribution, it increased the deposition strip, and in the spacing among beaks with C.V. of $10 \%$, with the addition of $0,1 \%$ of adjuvant and increasing in the pressure.
\end{abstract}

KEYWORDS: surfactant, application liquid, application technology.

\footnotetext{
${ }^{1}$ Eng $^{\mathrm{o}}$ Agrônomo, Pós-Graduando, Departamento de Engenharia Rural, UNESP, Jaboticabal - SP, felipetdacamara@ yahoo.com.br

${ }^{2}$ Biólogo, Pós-Graduando, Departamento de Fitossanidade, UNESP, Jaboticabal - SP.

${ }^{3}$ Eng $^{\mathrm{O}}$ Agrônomo, Pós-Graduando, Departamento de Fitossanidade, UNESP, Jaboticabal - SP.

${ }^{4}$ Eng $^{\circ}$ Agrônomo, Prof. Doutor, Departamento de Fitossanidade, UNESP, Jaboticabal - SP.

Recebido pelo Conselho Editorial em 31-1-2007
}

Aprovado pelo Conselho Editorial em: 22-11-2008 


\section{INTRODUÇÃO}

A avaliação do espectro de gotas é importante, pois, dependendo do alvo a ser controlado, exigem-se gotas de maior ou menor diâmetro, no qual o modelo de bico interfere, e também a sua distância em relação ao alvo, a pressão de pulverização, o ângulo de inclinação do bico, o tipo de calda utilizada na pulverização, entre outros fatores.

Outro aspecto a considerar é que gotas menores que $100 \mu \mathrm{m}$ são normalmente suscetíveis à deriva, muitas vezes perdendo-se no trajeto entre o pulverizador e o alvo desejado (CUNHA et al., 2004).

Em trabalhos para a caracterização do padrão de distribuição volumétrica, MATUO et al. (1994) relatam que cada tipo de bico tem um padrão de distribuição volumétrica diferente e que esse comportamento pode variar em função do espaçamento entre bicos e de sua distância em relação ao alvo, sendo essas características muito importantes no resultado da aplicação de produtos fitossanitários, interferindo na distribuição, deposição, cobertura e retenção de calda, com reflexos diretos sobre a eficácia do tratamento realizado.

A partir do padrão de distribuição de bicos individuais, pode-se simular a sobreposição de vários bicos, tal qual ocorre na barra de pulverização, que é expressa pelo coeficiente de variação obtido dessa análise, sendo indicado como ideal o valor de espaçamento entre bicos que resulte em C.V. de $10 \%$ (FAO, 1998).

A uniformidade do jato é importante para a adequada distribuição do produto na área-alvo, aumentando a possibilidade de controle da praga visada. É importante ressaltar que as pontas de pulverização de jato plano devem ser instaladas de modo que o jato aspergido forme ângulo fixo em relação à barra (entre 6 e $10^{\circ}$ ) para evitar o choque entre jatos de calda adjacentes, o que compromete a uniformidade de deposição (FERREIRA et al., 2007).

Para a realização desses estudos, é necessária mesa ou bancada de teste, na qual canaletas realizam a coleta do líquido a distâncias predeterminadas e depositam-no em recipientes individuais (CHAPPLE et al., 1993).

As especificações dos bicos de pulverização são definidas em testes com água, segundo norma da ASAE (2004), enquanto FAO (1998) recomenda o uso de água com 0,1\% de surfactante não-iônico, com temperatura entre 10 e $25^{\circ} \mathrm{C}$, e umidade relativa acima de $50 \%$.

Com relação ao acréscimo de surfactante, o padrão de distribuição das gotas e a vazão dos bicos tendem a ser diferentes do que quando se utiliza apenas água como líquido de aplicação (AZEVEDO, 2001).

O presente trabalho teve o objetivo de avaliar o espectro de gotas e o padrão de distribuição volumétrico de bico hidráulico de jato plano, modelo XR11003, em função do líquido de aplicação (água e água com 0,1\% de surfactante) e da pressão de pulverização (200 e $400 \mathrm{kPa}$ ).

\section{MATERIAL E MÉTODOS}

O trabalho foi conduzido em setembro de 2006, nas instalações do Departamento de Fitossanidade da Faculdade de Ciências Agrárias e Veterinárias - Câmpus de Jaboticabal - SP, UNESP, constando de dois experimentos distintos, sendo um de análise do espectro de gotas e outro dos perfis de distribuição volumétrica, ambos utilizando-se de bicos hidráulicos XR11003 de jato plano.

A análise do espectro de gotas foi realizada de forma direta, utilizando-se de analisador de partículas (Mastersizer $\mathrm{S} \AA$, Malvern Instruments Co.), lente focal de $300 \mathrm{~mm}$, comprimento de onda de laser de 0,5 a $900 \mathrm{~nm}$, que se baseia na difração da luz após o choque dessa com as gotas, em que o diâmetro das gotas é proporcional ao ângulo do desvio sofrido pelo raio laser. $\mathrm{O}$ bico de pulverização foi instalado a $40 \mathrm{~cm}$ do feixe de laser, e foi movimentado $45^{\circ}$ para a direita e para a esquerda com o intuito de que todo o jato atravessasse o feixe. Para manter a pressão constante, 
utilizou-se de ar comprimido controlado com regulador de pressão de precisão, operado manualmente, munido de manômetro analógico calibrado para a função. $\mathrm{O}$ acionamento do fluxo de calda e do mecanismo de movimentação do bico é realizado simultaneamente por meio de interruptor elétrico que comanda a válvula solenoide do circuito hidráulico e mecanismo de oscilação do bico.

As condições ambientais médias no momento de realização dos experimentos foram: temperatura do ar de $27^{\circ} \mathrm{C}$; umidade relativa do ar de 58\%; ausência de ventos e luminosidade externa, uma vez que as análises foram realizadas com as luzes apagadas.

A decodificação dos dados, segundo o algoritmo elaborado para a caracterização do diâmetro de partículas por difração de raios laser, foi realizada pelo software Mastersizer S V.2.19, Malvern Instruments Co. Os valores relacionados ao espectro de tamanho de partículas são processados e tabelados diretamente pelo software, sendo apresentados de forma gráfica ou tabela, conforme configuração determinada pelo usuário.

Este experimento teve o objetivo de avaliar o diâmetro de gotas, tal que $10 \%$ do volume aplicado seja inferior a esse valor $\left(\mathrm{DV}_{0,1}\right)$; o diâmetro de gotas, tal que $50 \%$ do volume aplicado seja inferior a esse valor $\left(\mathrm{DV}_{0,5}\right)$; o diâmetro de gotas, tal que $90 \%$ do volume aplicado seja inferior a esse valor $\left(\mathrm{DV}_{0,9}\right)$; a amplitude relativa $(\mathrm{AR})$ e a percentagem do volume aplicado cujas gotas possuam diâmetro inferior a $100 \mu \mathrm{m}(\mathrm{DV}<100 \mu \mathrm{m})$.

O delineamento foi o inteiramente casualizado, em esquema fatorial $2 \times 2 \times 3$, com três repetições, em que o primeiro fator representa o líquido de aplicação (L1 = água e L2 = água + $0,1 \%$ de surfactante Haiten), o segundo representa a pressão de pulverização (P1 = $200 \mathrm{kPa}$ e P2 = $400 \mathrm{kPa}$ ) e o terceiro representa três bicos hidráulicos XR11003 (B1, B2 e B3).

Os dados obtidos foram tabulados e submetidos à análise de variância e ao teste de comparação de médias de Tukey, a 5\% de probabilidade (BANZATTO \& KRONKA, 1995).

O experimento para a determinação do perfil de distribuição volumétrica da calda pulverizada pela ponta XR11003 foi realizado em uma mesa de provas construída de acordo com as especificações da ORGANIZACIÓN MUNDIAL DE LA SALUD (1976) para testes de bicos, constituída de 67 canaletas de metal corrugado com 2,5 cm de largura cada, totalizando $167,5 \mathrm{~cm}$ de largura por $100 \mathrm{~cm}$ de comprimento. As canaletas possuem inclinação de $15^{\circ}$ para o líquido pulverizado escoar até tubos de ensaio graduados, utilizados como coletores.

O bico hidráulico XR11003 de jato plano foi posicionado verticalmente à altura da mesa de $50 \mathrm{~cm}$, acima da canaleta $\mathrm{n}^{\circ} 33$, no centro da mesa de provas.

As condições ambientais no momento do ensaio foram: temperatura do ar de 23 a $27{ }^{\circ} \mathrm{C}$, umidade relativa do ar de 54 a 58\%, com ausência de ventos.

Para a determinação do perfil de distribuição volumétrica, o tempo de coleta de cada bico hidráulico foi de 120 segundos, sob pressão constante de $200 \mathrm{kPa}$, e 90 segundos, sob pressão constante de $400 \mathrm{kPa}$, pois, nessa segunda pressão, o tempo de 120 segundos emitiria maior volume do que o suportado pelos coletores.

O ensaio teve por objetivo avaliar a faixa de deposição individual (FDI), o ângulo real do jato de pulverização, o espaçamento entre bicos com C.V. de $10 \%$ (Esp) e a percentagem de sobreposição.

A FDI foi obtida verificando-se, nos coletores, o número de canaletas que continha o líquido após a pulverização, multiplicando-se pela distância entre canaletas $(2,5 \mathrm{~cm})$.

O ângulo do jato $(\alpha)$ foi calculado pela relação entre a altura do bico (A) e a FDI, pela eq.(1):

$\alpha=\{$ arctangente $[(\mathrm{FDI} / 2) / \mathrm{A}]\} 2$ 
O Esp foi calculado simulando-se vários espaçamentos entre bicos e calculando-se o C.V. do resultado da sobreposição; com isso, ao obter-se espaçamento com C.V. próximo a $10 \%$, foi determinado o C.V. para quatro espaçamentos menores e quatro maiores do que esse, reduzindo e aumentando $2,5 \mathrm{~cm}$ a esse valor, construindo-se, em seguida, um gráfico com a equação de regressão polinomial de segundo grau, a partir da qual foi determinado o Esp. a eq.(2):

A percentagem de sobreposição (S) foi obtida pela relação entre o Esp e a FDI, de acordo com

$$
\mathrm{S}=100[1-(\mathrm{Esp} / \mathrm{FD})]
$$

Para determinar a vazão total do bico, coletou-se o líquido num período de 30 segundos, transformando-o para $\mathrm{L} \mathrm{min}^{-1}$.

Foi utilizado o delineamento experimental inteiramente casualizado, no esquema fatorial $2 \times 2$, com três repetições, em que o primeiro fator representa o líquido de aplicação (L1 = água e $\mathrm{L} 2=$ água $+0,1 \%$ de surfactante) e o segundo representa a pressão de pulverização $(\mathrm{P} 1=200 \mathrm{kPa}$ e $\mathrm{P} 2=400 \mathrm{kPa})$.

\section{RESULTADOS E DISCUSSÃO}

Na Tabela 1, as médias seguidas de mesma letra maiúscula em cada coluna não diferem entre si, pelo Teste de Tukey, a 5\% de probabilidade. Nas colunas com ausência de letras, houve interação significativa entre dois ou mais fatores, cujo desdobramento será explicado na tabela de desdobramento da interação.

TABELA 1. Síntese da análise de variância e do teste de médias para as variáveis $\mathrm{DV}_{0,1}, \mathrm{DV}_{0,5}$, $\mathrm{DV}_{0,9}, \mathrm{AR}$ e $\mathrm{DV}<100 \mu \mathrm{m}$. Summary of analysis of variance and test averages for the variables $\mathrm{DV}_{0,1}, \mathrm{DV}_{0,5}, \mathrm{DV}_{0,9}, \mathrm{AR}$ e $\mathrm{DV}<100 \mu \mathrm{m}$.

\begin{tabular}{|c|c|c|c|c|c|}
\hline Fatores & $\begin{array}{c}\mathrm{DV}(0,1) \\
(\mu \mathrm{m})\end{array}$ & $\begin{array}{c}\mathrm{DV}(0,5) \\
(\mu \mathrm{m})\end{array}$ & $\begin{array}{c}\mathrm{DV}(0,9) \\
(\mu \mathrm{m})\end{array}$ & $\mathrm{AR}$ & $\begin{array}{c}\mathrm{DV}<100 \mu \mathrm{m} \\
\%\end{array}$ \\
\hline \multicolumn{6}{|c|}{ Líquido (L) } \\
\hline L1 & $72,11 \mathrm{~B}$ & 157,95 & 297,23 & $1,439 \mathrm{~A}$ & $22,02 \mathrm{~A}$ \\
\hline L2 & $77,01 \mathrm{~A}$ & 156,22 & 293,90 & $1,373 \mathrm{~B}$ & $20,04 \mathrm{~B}$ \\
\hline \multicolumn{6}{|c|}{ Pressão (P) } \\
\hline P1 & $83,96 \mathrm{~A}$ & 170,99 & 325,08 & $1,410 \mathrm{~A}$ & 16,02 B \\
\hline P2 & $65,16 \mathrm{~B}$ & 143,19 & 266,05 & $1,403 \mathrm{~A}$ & $26,04 \mathrm{~A}$ \\
\hline \multicolumn{6}{|l|}{$\operatorname{Bico}(\mathrm{B})$} \\
\hline B1 & $75,33 \mathrm{~A}$ & $154,95 \quad$ В & $284,31 \mathrm{~B}$ & $1,348 \mathrm{~B}$ & $20,82 \mathrm{~A}$ \\
\hline B2 & $74,44 \mathrm{~A}$ & $157,79 \mathrm{AB}$ & 297,77 A & $1,416 \mathrm{~A}$ & $21,02 \mathrm{~A}$ \\
\hline B3 & $73,90 \mathrm{~A}$ & $158,53 \mathrm{~A}$ & $304,62 \mathrm{~A}$ & $1,454 \mathrm{~A}$ & $21,24 \mathrm{~A}$ \\
\hline \multicolumn{6}{|l|}{ Teste F } \\
\hline $\mathrm{L}$ & $71,57 * *$ & $3,33^{\mathrm{NS}}$ & $1,46^{\mathrm{NS}}$ & $24,91 * *$ & $36,63 * *$ \\
\hline $\mathrm{P}$ & $1.055,31 * *$ & $858,91 * *$ & $457,65 * *$ & $0,28^{\mathrm{NS}}$ & $935,07 * *$ \\
\hline $\mathrm{B}$ & $2,06^{\mathrm{NS}}$ & $5,30 *$ & $18,68 * *$ & $22,15 * *$ & $0,54^{\mathrm{NS}}$ \\
\hline LxP & $2,05^{\mathrm{NS}}$ & $7,63 *$ & $6,70 *$ & $2,58^{\mathrm{NS}}$ & $0,47^{\mathrm{NS}}$ \\
\hline $\mathrm{LxB}$ & $0,15^{\mathrm{NS}}$ & $0,07^{\mathrm{NS}}$ & $1,67^{\mathrm{NS}}$ & $2,73^{\mathrm{NS}}$ & $0,40^{\mathrm{NS}}$ \\
\hline $\mathrm{PxB}$ & $1,85^{\mathrm{NS}}$ & $0,03^{\mathrm{NS}}$ & $0,24^{\mathrm{NS}}$ & $0,69^{\mathrm{NS}}$ & $0,81^{\mathrm{NS}}$ \\
\hline LxPxB & $1,10^{\mathrm{NS}}$ & $0,51^{\mathrm{NS}}$ & $0,02^{\mathrm{NS}}$ & $0,04^{\mathrm{NS}}$ & $0,06^{\mathrm{NS}}$ \\
\hline C.V.\% & 2,33 & 1,81 & 2,80 & 2,82 & 4,67 \\
\hline
\end{tabular}

Em cada coluna, para cada fator, médias seguidas de mesmas letras maiúsculas não diferem entre si, pelo teste de Tukey, a 5\% de probabilidade; **: significativo $(\mathrm{P}<0,01)$; *: significativo $(\mathrm{P}<0,05)$; ${ }^{\mathrm{NS}}$ : não-significativo; C.V.: coeficiente de variação 
Observa-se, na Tabela 1 , que o $\mathrm{DV}_{0,1}$ foi maior quando se utilizou como líquido de pulverização água $+0,1 \%$ de surfactante (Haiten) e com pressão de pulverização de $200 \mathrm{kPa}$, sendo que, quanto maior for esse valor, menor é o risco de deriva, significando que o uso de surfactante diminuiu o risco de deriva na pulverização. Outro ponto importante foi que não houve diferença entre os três bicos avaliados quanto ao $\mathrm{DV}_{0,1}$. Porém, analisando-se o $\mathrm{DV}_{0,5}$ e $\mathrm{DV}_{0,9}$, verifica-se que o bico 3 apresentou os maiores valores, e o bico 1, os menores. A variação observada pode ser considerada baixa visto os processos envolvidos na fabricação do bico. A diferença estatística deu-se pela elevada possibilidade de reprodução do tratamento com o método utilizado.

Ainda na Tabela 1, nota-se que o uso de surfactante diminuiu a AR, indicando menor variação no espectro de tamanho de gotas. Nessa variável, a pressão de pulverização não influenciou e houve diferenças entre os bicos avaliados.

Segundo CUNHA et al. (2004), gotas com diâmetro inferior a $100 \mu \mathrm{m}$ são as principais responsáveis pelo aumento no risco da deriva. Sendo assim, a adição de surfactante à calda, na pressão de $200 \mathrm{kPa}$, diminuiu a percentagem de gotas menores que $100 \mu \mathrm{m}$, reduzindo o risco de deriva.

$\mathrm{Na}$ Tabela 2, verifica-se que o aumento na pressão diminuiu o valor $\operatorname{deDV}_{0,5}$, e que para a pressão de $400 \mathrm{kPa}$, o uso de surfactante proporcionou maior valor, enquanto na pressão de $200 \mathrm{kPa}$, não houve diferença significativa.

TABELA 2. Interação entre os fatores Líquido de aplicação e pressão de pulverização para a variável $\mathrm{DV}_{0,5}$. Interaction between the factors of sprayed liquid and application pressure for variable $\mathrm{DV}_{\mathbf{0 , 5}}$.

\begin{tabular}{lcc}
\hline \multirow{2}{*}{ Líquido (L) } & \multicolumn{2}{c}{ Pressão (P) } \\
\cline { 2 - 3 } & P1 - 200 kPa & P2 - 400 kPa \\
\hline L1 - Água & $171,43 \mathrm{~A} \mathrm{a}$ & $141,01 \mathrm{~B} \mathrm{~b}$ \\
L2 - Água $+0,1 \%$ de surfactante & $170,54 \mathrm{~A} \mathrm{a}$ & $145,36 \mathrm{~B} \mathrm{a}$ \\
\hline
\end{tabular}

Médias seguidas de mesma letra minúscula nas colunas e maiúscula nas linhas não diferem entre si, pelo Teste de Tukey, a 5\% de probabilidade.

$\mathrm{Na}$ Tabela 3, observa-se maior valor do $\mathrm{DV}_{0,9}$ para a pressão de $200 \mathrm{kPa}$; com a adição de surfactante, houve menor valor para a pressão de $200 \mathrm{kPa}$ e não diferiu, na pressão de $400 \mathrm{kPa}$, do tratamento com água.

TABELA 3. Interação entre os fatores Líquido de aplicação e pressão de pulverização para a variável $\mathrm{DV}_{0,9}$. Interaction between the factors of sprayed liquid and application pressure for variable $\mathrm{DV}_{0,9}$.

\begin{tabular}{lcc}
\hline \multirow{2}{*}{ Líquido (L) } & \multicolumn{2}{c}{ Pressão (P) } \\
\cline { 2 - 3 } & $\mathrm{P} 1-200 \mathrm{kPa}$ & $\mathrm{P} 2-400 \mathrm{kPa}$ \\
\hline L1 - Água & $330,32 \mathrm{~A} \mathrm{a}$ & $264,14 \mathrm{~B} \mathrm{a}$ \\
L2 - Água $+0,1 \%$ de surfactante & $319,84 \mathrm{~A} \mathrm{~b}$ & $267,96 \mathrm{~B} \mathrm{a}$ \\
\hline
\end{tabular}

Médias seguidas de mesma letra minúscula nas colunas e maiúscula nas linhas não diferem entre si, pelo Teste de Tukey, a 5\% de probabilidade.

A distribuição das gotas por classe de diâmetro está apresentada na Figura 1. Observa-se distribuição regularmente uniforme quando se utilizou o surfactante. Quando foi utilizada água, o bico 2 apresentou irregularidade próxima aos diâmetros de 200 e entre 350 e $450 \mu \mathrm{m}$, na pressão de $200 \mathrm{kPa}$, e os bicos 1 e 3 na pressão de $400 \mathrm{kPa}$, entre 350 e $450 \mu \mathrm{m}$.

Essas irregularidades podem ser devidas à interação entre a tensão superficial da água e a pressão de trabalho. Isso pode indicar a realização do teste com um surfactante mais adequado, conforme indicado pela FAO (1998), além de aproximar-se mais das condições de trabalho no campo. 

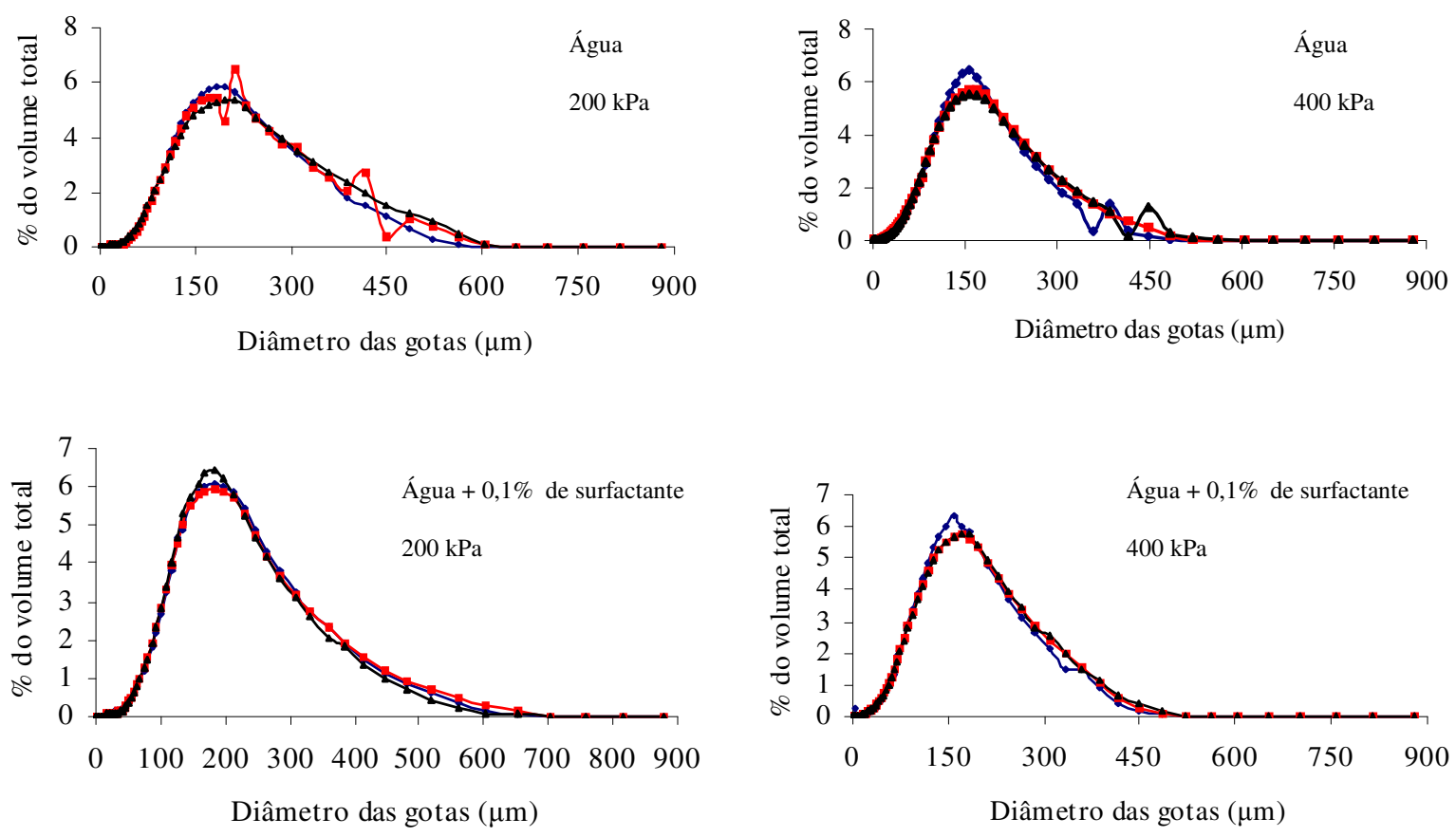

FIGURA 1. Espectro de gotas pulverizadas com os bicos hidráulicos 1 (४), 2 (๘) e 3 (४).Spectrum of drops sprayed with hydraulic nozzles $1(\diamond), 2$ ( $\square)$ e $3(\Delta)$.

Observa-se, na Figura 2, o espaçamento entre bicos em função do coeficiente de variação, apenas para os coeficientes de variação mais próximos de $10 \%$, e a equação de regressão para a determinação do espaçamento entre bicos, em função do coeficiente de variação.
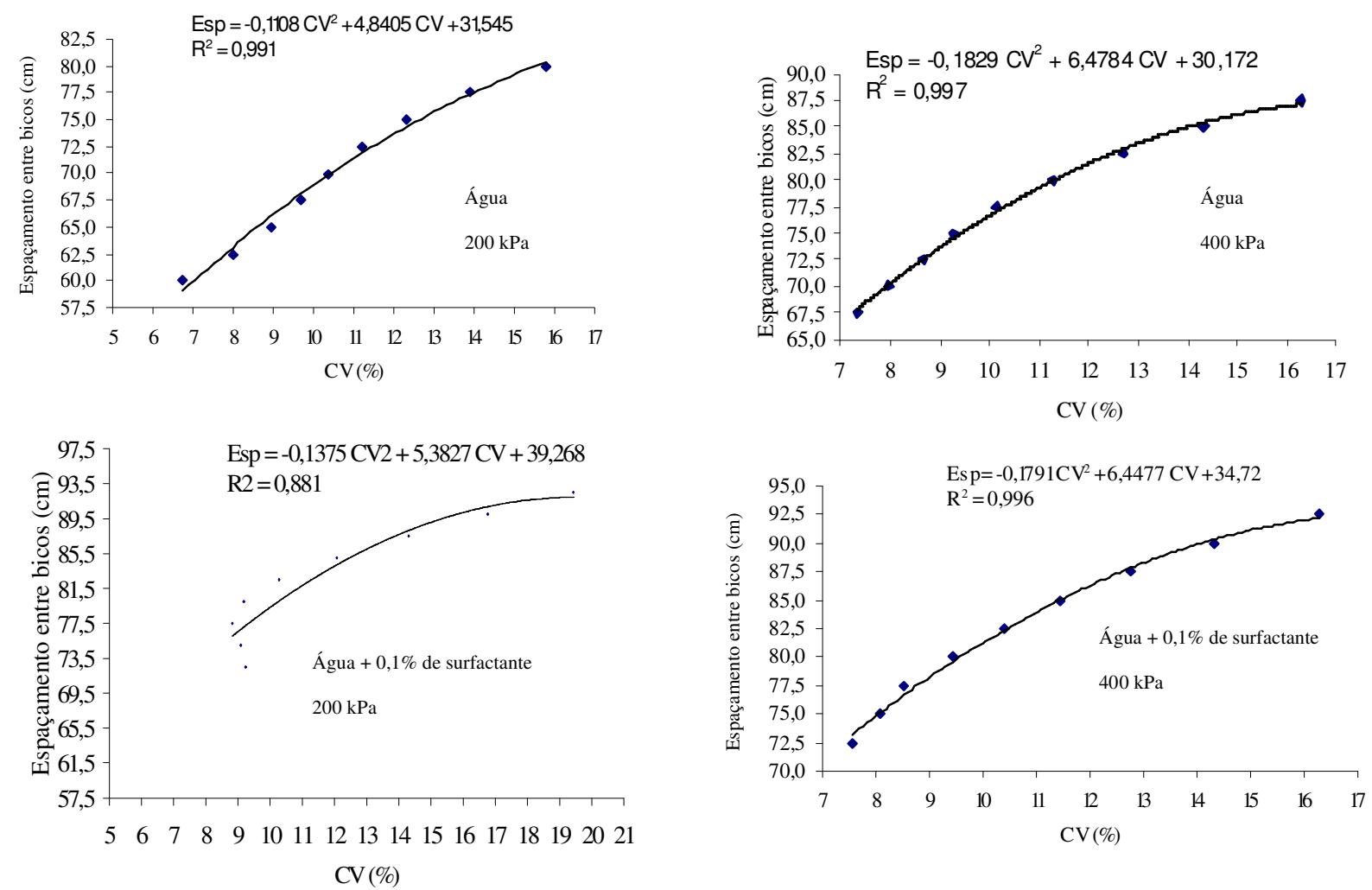

FIGURA 2. Espaçamento entre bicos em função do coeficiente de variação. Spacing between nozzles according to the coefficient of variation. 
Os resultados da distribuição volumétrica estão apresentados na Tabela 4, na qual se nota que o ângulo do jato de pulverização variou pouco do indicado para o bico ensaiado $\left(110^{\circ}\right)$, sendo maior para a pressão de $400 \mathrm{kPa}$ e com o uso de surfactante. Resultados semelhantes foram observados por FERNANDES et al. (2007). Os autores verificaram que o ângulo de pulverização de pontas XR $110015 \mathrm{VS}$, a $0,50 \mathrm{~m}$ da altura foi, respectivamente, $110^{\circ}$ e $115^{\circ}$ para as pressões de 200 e $300 \mathrm{kPa}$, resultando em pequena diferença nas larguras da faixa de deposição. Contudo, a distribuição do jato de pulverização praticamente não se alterou.

TABELA 4. Ângulo do jato de pulverização, vazão por bico, faixa de deposição individual (FDI), espaçamento entre bicos com C.V. de $10 \%$ (Esp) e percentagem de sobreposição, utilizando-se de dois tipos de líquidos de aplicação, em duas pressões de trabalho. Angle of the jet spray, flow nozzles, track individual deposition (FDI), spacing between nozzles with $\mathrm{CV}$ of $10 \%$ (Esp) and percentage of overlap, using two types of liquids for application in two pressures of work.

\begin{tabular}{ccccccc}
\hline $\begin{array}{c}\text { Líquido de } \\
\text { Aplicação }\end{array}$ & $\begin{array}{c}\text { Pressão } \\
(\mathrm{kPa})\end{array}$ & $\begin{array}{c}\text { Ângulo } \\
\left({ }^{\circ}\right)\end{array}$ & $\begin{array}{c}\text { Vazão } \\
\mathrm{L} \mathrm{min}^{-1}\end{array}$ & $\begin{array}{c}\text { FDI } \\
(\mathrm{cm})\end{array}$ & $\begin{array}{c}\text { Esp } \\
(\mathrm{cm})\end{array}$ & $\begin{array}{c}\text { Sobreposição } \\
(\%)\end{array}$ \\
\hline \multirow{2}{*}{ Água } & 200 & 106 & 0,9 & 132,5 & 69 & 48 \\
\cline { 2 - 7 } & 400 & 111 & 1,33 & 145 & 77 & 47 \\
\hline \multirow{2}{*}{ Água + Surfactante } & 200 & 109 & 0,92 & 140 & 79 & 44 \\
\cline { 2 - 7 } & 400 & 114 & 1,33 & 155 & 81 & 48 \\
\hline
\end{tabular}

Verifica-se que a vazão foi maior com o aumento na pressão e praticamente não variou com a adição de $0,1 \%$ de surfactante (Tabela 4); houve aumento na faixa de deposição individual (FDI) e espaçamento entre bicos para o C.V. de $10 \%$ (Esp) com a adição de surfactante e aumento na pressão. BAUER \& RAETANO (2004) encontraram na pressão de $200 \mathrm{kPa}$ valor de $170 \mathrm{~cm}$ para FDI, bem maior do que foi encontrado neste trabalho, e $65 \mathrm{~cm}$ para Esp, valor pouco menor do que o observado neste trabalho. A percentagem de sobreposição entre os jatos de pulverização foi pouco alterada entre os tratamentos, ficando em torno de $45 \%$, valor bem acima do que os $30 \%$ recomendados pelos fabricantes.

Nas Figuras 3 e 4, observa-se que houve maior volume de líquido na pressão de $400 \mathrm{kPa}$ nas canaletas mais afastadas e menor na canaleta central, comparado com a pressão de $200 \mathrm{kPa}$, tanto para os tratamentos com água quanto nos com água $+0,1 \%$ de surfactante. Verifica-se, ainda, que a adição de surfactante interfere pouco na distribuição volumétrica para a pressão de $400 \mathrm{kPa}$, enquanto, na pressão de $200 \mathrm{kPa}$, verifica-se pior distribuição volumétrica com a adição de surfactante. FERNANDES et al. (2007) verificaram que as diferentes pressões resultaram em aumento de $19,4 \%$, passando de 0,258 para $0,308 \mathrm{~L} \mathrm{~min}^{-1}$, respectivamente, para as pressões de 200 e $300 \mathrm{kPa}$. Apesar da diferença na vazão, não se verificou alteração significativa na curva de deposição para as pressões analisadas. 

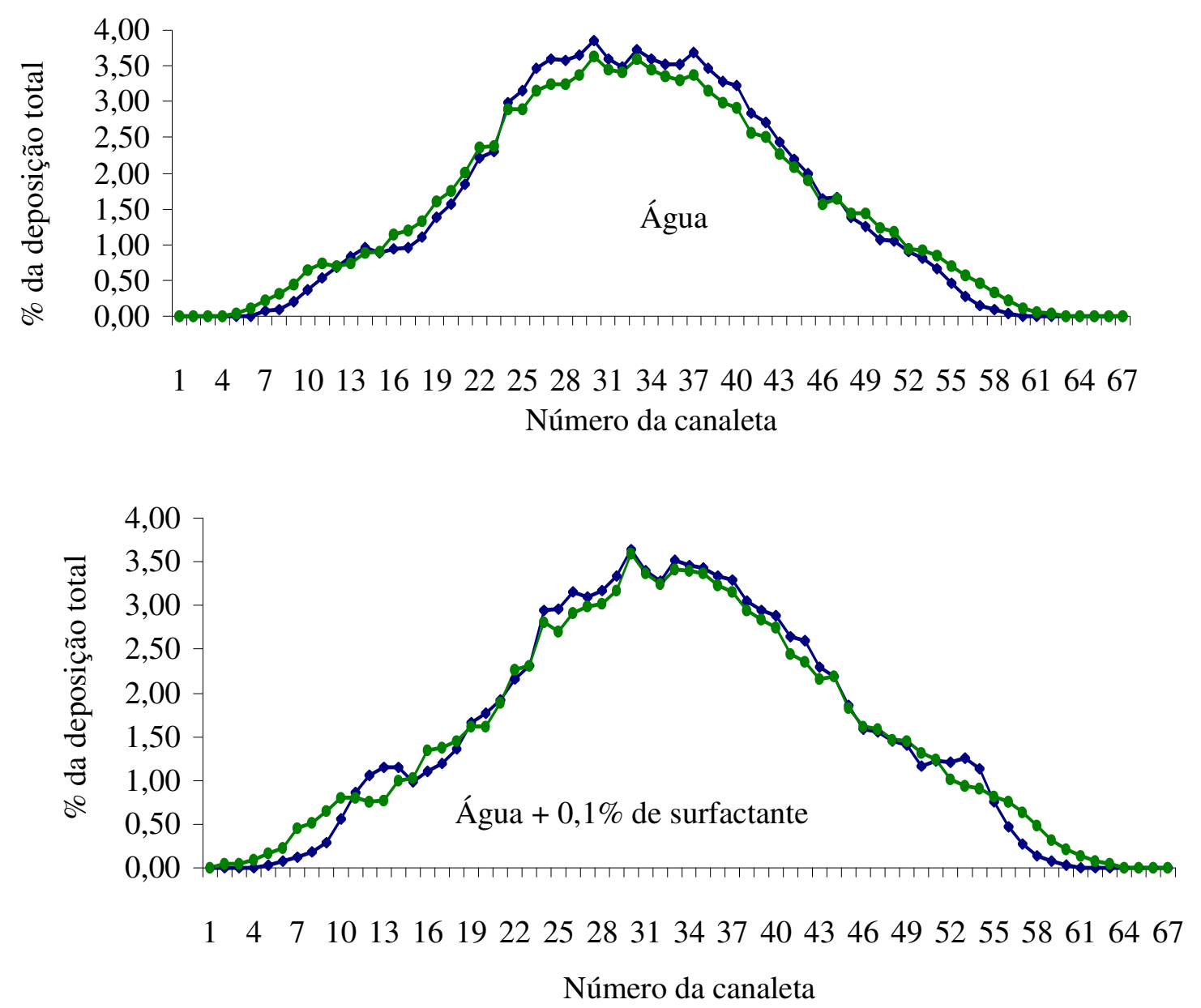

FIGURA 3. Perfil de distribuição volumétrica nas pressões de $200 \mathrm{kPa}(\bullet)$ e $400 \mathrm{kPa}(\bullet)$, utilizando-se de água e água $+0,1 \%$ de surfactante. Profile of distribution volume at pressures of $200 \mathrm{kPa}(\bullet)$ and $400 \mathrm{kPa}(\bullet)$, using water and water $+0.1 \%$ of surfactant. 


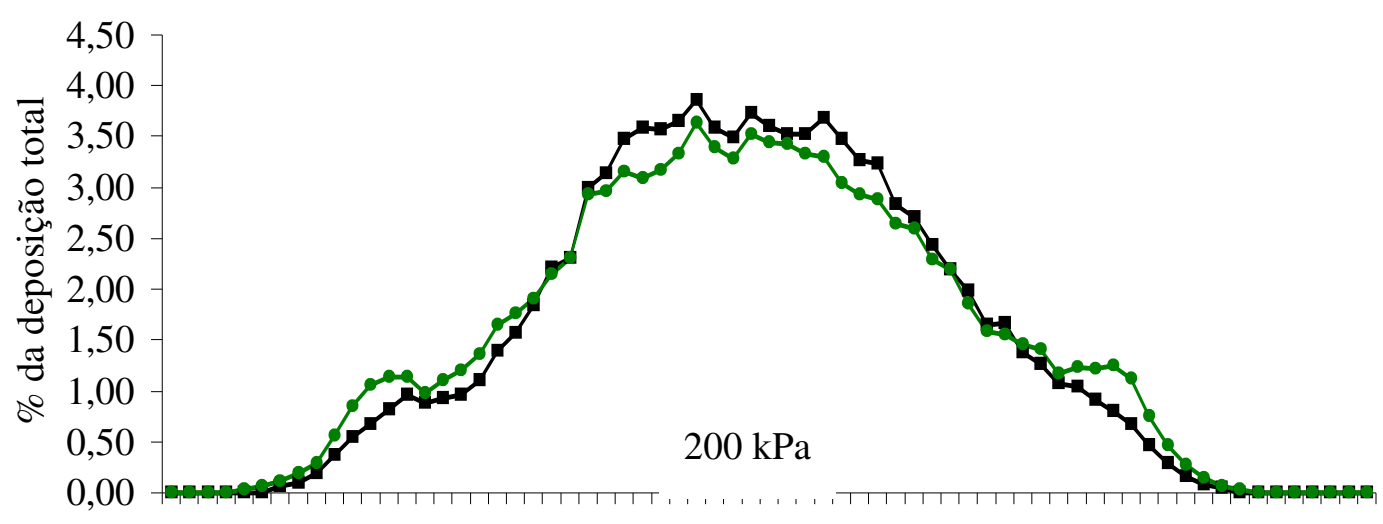

$144 \quad 7 \quad 1013161922252831343740434649525558616467$

Número da canaleta

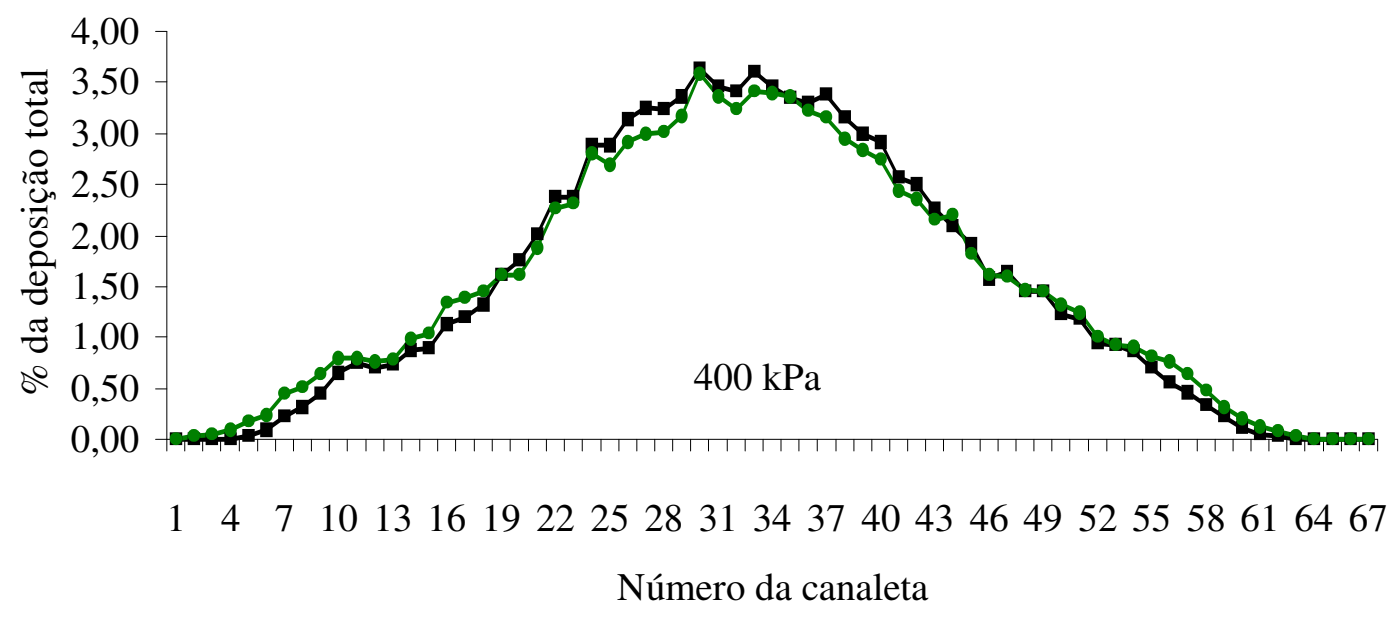

FIGURA 4. Perfil de distribuição volumétrica na pressão de 200 e $400 \mathrm{kPa}$, utilizando-se, como líquido de aplicação, de água (ロ) e água $+0,1 \%$ de surfatante $(\bullet)$. Profile of distribution volume in the pressure of 200 and $400 \mathrm{kPa}$, using water as profit for the application $(\square)$ and water $+0.1 \%$ of surfactant $(\bullet)$.

\section{CONCLUSÕES}

O diâmetro de gotas foi maior quando se utilizou a pressão de $200 \mathrm{kPa}$ e surfactante. A percentagem de gotas com diâmetro inferior a $100 \mu \mathrm{m}$ foi menor com o uso de surfactante e para a menor pressão utilizada $(200 \mathrm{kPa})$.

Ocorreu menor amplitude relativa com o uso de surfactante, enquanto a pressão de pulverização não influenciou e houve diferenças entre os exemplares dos bicos avaliados.

Para os perfis de distribuição volumétrica, ocorreu aumento na faixa de deposição e no espaçamento entre bicos com C.V. de $10 \%$ com a adição de surfactante e aumento na pressão.

\section{REFERÊNCIAS}

ASAE. AMERICAN SOCIETY OF AGRICULTURAL ENGINEERING. Spray nozzle classification by droplet spectra. St. Joseph: ASAE, 2004. p.411-14. (ASAE Standard S572 FEB04). 
AZEVEDO, L.A.S. Proteção integrada de plantas com fungicida. São Paulo: Luiz Azevedo, 2001. $230 \mathrm{p}$.

BANZATTO, D.A.; KRONKA, S.N. Experimentação agrícola. Jaboticabal: FUNEP, 1995. $247 \mathrm{p}$.

BAUER, F.C.; RAETANO, C.G. Perfis de distribuição volumétrica de pontas XR11003 E TXVK-4 em diferentes condições de pulverização. Engenharia Agrícola, Jaboticabal, v.24, n.2, p.364-73, 2004.

CHAPPLE, A.C.; HALL, F.R.; BISHOP, B.L. Assesment of single nozzle patternation and extrapolation to moving booms. Crop Protection, Guildford, v.12, n.1, p.207-13, 1993.

CUNHA, J.P.A.; TEIXEIRA, M.M.; VIEIRA, R.F.; FERNANDES, H.C.; COURY, J.R. Espectro de gotas de bicos de pulverização hidráulicos de jato plano e de jato cônico vazio. Pesquisa Agropecuária Brasileira, Brasília, v.39, n.10, p.977-85, 2004.

FAO. FOOD AND AGRICULTURE ORGANIZATION OF THE UNITED NATIONS. Agricultural pesticide sprayers. Rome, 1998. v.2, p.62.

FERNANDES, A.P.; PARREIRA, R.S.; FERREIRA, M.C.; ROMANI, G.N. Caracterização do perfil de deposição e do diâmetro de gotas e otimização do espaçamento entre bicos na barra de pulverização. Engenharia Agrícola, Jaboticabal, v.27, n.3, p.728-33, 2007.

FERREIRA, M.C.; COSTA, G.M.; SILVA, A.R.; TAGLIARI, S.R.A. Fatores qualitativos da ponta de energia hidráulica Adga 110015 para pulverização agrícola. Engenharia Agrícola, Jaboticabal, v.27, n.2, p.471-8, 2007.

MATUO, T.; PERESSIN, V.A.; PERECIN, D.; PIO, L.C.; BRAZ, B.A. Aplicação em jato dirigido em cana-de-açúcar. I. Espaçamento entre bicos twinjet para aplicação do herbicida MSMA.

Engenharia Agrícola, Jaboticabal, v.14, p.69-82, 1994.

ORGANIZACIÓN MUNDIAL DE LA SALUD. Material de lucha contra los vetores. Genebra, 1976. $189 \mathrm{p}$. 